\section{Grain Sizing of Anodized Aluminum by Color Image Analysis}

\section{Sylvain Laroche \& Clement Forget,Clemex Technologies}

Grain size characterization in Aluminum alloys can be correlated with thermo-mechanical processing properties'. In order to predict the processing characteristics of these alloys under certain combinations of strain, deformation and temperature, the metallographic measure of the grain size can be used. Most of the techniques that have been proposed so far do not provide reliable and reproducible quantitative metallographic measurements of the grain size due to human error. Considering that this manual task is also tedious to perform, a general color image analysis algorithm is proposed to automate the characterization process using an optical microscope with polarized light. This algorithm was tested on several ingots and on rolled aluminum samples. The results show robustness in several conditions, even when the grains can barely be seen by a human operator. Other image analysis techniques have been proposed but where judged too slow or too complex, particularly when gathering data over several fields. Time constraints specific to industrial seffings were taken into account when implementing a new algorithm. A complete characterization of the entire surface of an ingot can be obtained within a reasonable time limit. The same algorithm was tested with other types of color material. This general framework for handling segmentation of color grain fulfills industrial requirements for the characterization of material seen under a microscope with polarized light.

\section{Introduction}

Several image analysis techniques have been considered for segmentation of color images. However, they tend to focus on the specificity of a particular application ${ }^{234}$. Having this consideration in mind, an approach is proposed based on a general mathematical morphology

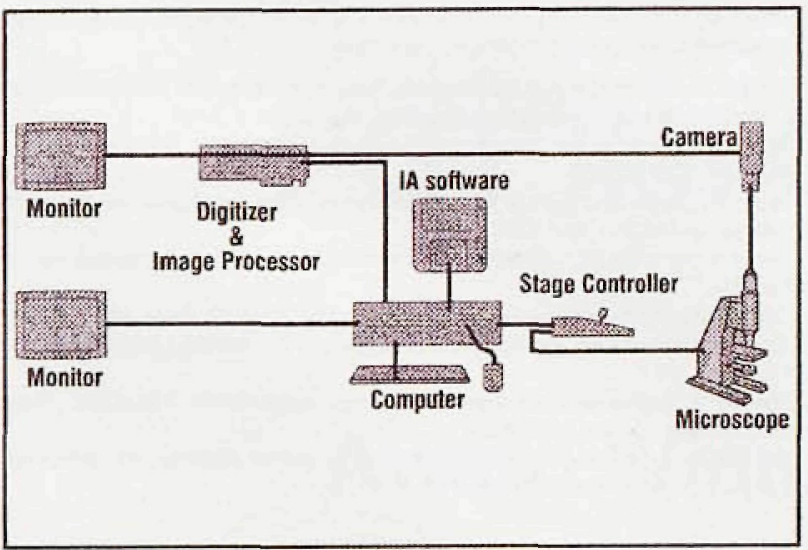

Fig.1 Image analysis system

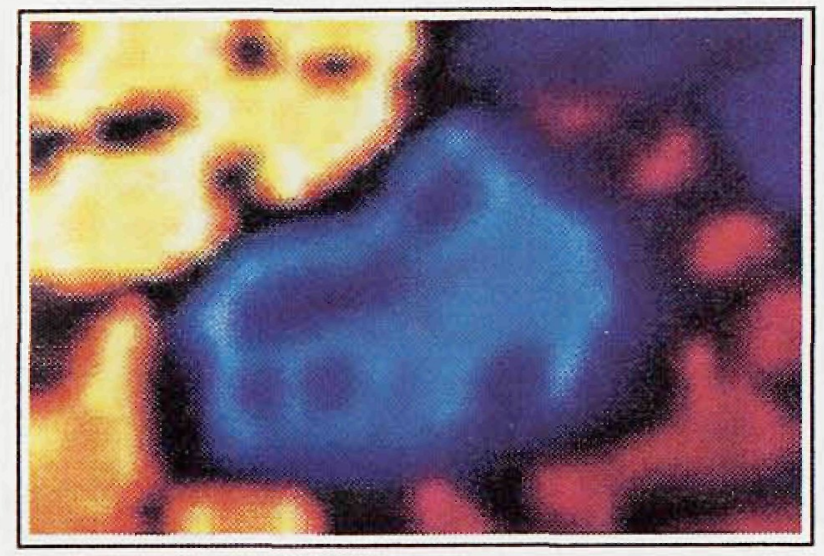

Fig. 3 Close-up of a grain framework. Most of the applications solved by mathematical morphology involve the analysis of binary or gray tone images ${ }^{5,6}$. Basic transforms such as erosion, dilation, opening, closing, gradient, etc., do not have a clear counterpart in any color space. The algorithm developed for this project is based on a set of morphological transforms into the HSI (Hue/Saturation/Intensity) color space. This space offers a natural basis to make color judgments while keeping the intensity (brightness) information separate

The hue value of a pixel refers to its spectrai color. One approach has recommended that a color image be segmented into different regions via grayscale thresholding of the hue component. However, hue can have singularities for pixels at very low saturation (gray pixels). The method from [3] entails that hue be set arbitrarily for these pixels. By contrast, the technique proposed below overcomes this inadequacy by considering HuelSaturation as an interdependent pair.

\section{Method}

An integrated image analysis system, combining a general vision software, a high speed image processing board and all controls for the microscope (stage autofocus, etc.) was used to develop the color segmentation algorithm. The system featured a dual monitor configuration to present the graphical user interface (GUI) and the color image (figure 1).

Each field of an Al ingot, seen with a polarizer at magnification of $40 \mathrm{X}$, was digitized from a triple CCD color camera into an RGB 24 bit frame buffer and converted into the HSI format by the high speed processing board. A color shading correction compensated for the variations in lighting conditions over the field of view. The system was calibrated - each pixel representing 3 microns. One digitized field covered 2.77 square $\mathrm{mm}$. Autofocus was automated and controlled by the system. A typical image is shown in figure 2.

Different ingots or rolled samples were scanned in sequence generating over 200 fields. Each field was processed using the color segmentation algorithm

\section{Continued on Page 18}

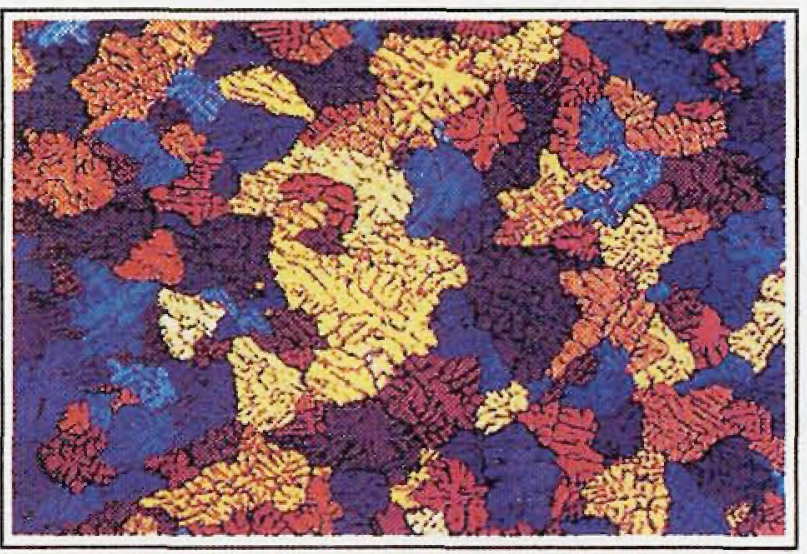

Fig.2 Typical image

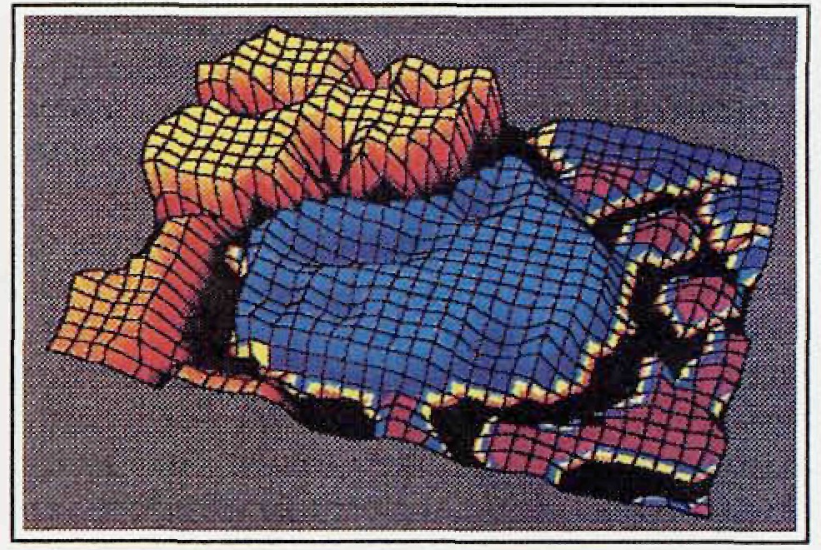

Fig. 4 3D representation of same grain 
Color Image Analysis - Continued from page 17

and all measures were gathered for each grain immediately after the segmentation process was applied to a field. The operator visualized the statistical results afterwards and easily reviewed all requested measures as well as the boundary of any segmented grain. This was possible since the location of each grain in the sample was mapped and saved in memory.

\section{Mathematical Morphology into the HSI space}

The RGB color model is inadequate for image analysis since the linear combination of the three components (RGB) is not easily deduced for expressing a region with similar colors. The HSI color space is technically more suitable and is closer to the way we express color in our everyday lives.

The basic transforms of mathematical morphology were implemented into the HSI space. Color erosion, dilation, opening, closing, thresholding gradient were available in the general vision software. The implementation of such transforms was complicated by the non-linear features of the HSI space. For instance, eroding only the intensity component was not sufficient to properly control the propagation of hue and saturation inside the neighborhood covered by the structuring element. Further, dilating only the intensity of a color grain to fill up the dark gaps between adjacent neighbors could not propagate the HS pair properly over the surrounding area. The proposed implementation deals with this important problem, in particular during the prefiltering procedure that aims at removing the gaps between grains before the color gradient is applied.

\section{Color Segmentation Algorithm}

Difficult segmentation problems were handled by watershed algorithms applied on gray tone images ${ }^{7,8}$. A successful solution can be achieved based on this technique if proper prefiltering is performed to deal with the high noise content of a gradient image.

The original aspects of the implementation are mainly:

1) The dark gaps (figures $3 \& 4$ ) are filled by a color morphological closing before performing the color gradient. The result of closing appears in figure 5 .

2) The gradient is improved by using the coarse markers that result in a single watershed per grain (figure 6). Further, figures 7 and 8 show how a region is defined for the HS pair when thresholding. This is done to obtain the coarse markers corresponding to a single color range.

3) The complete segmentation is carried out after the separation of each watershed/grain is thinned to a width of one pixel (figures 9 \& 10).

4) Several measures such as ASTM-112 can be computed before presenting the final statistics cumulated over the entire sample. It is also possible to review the segmentation of each grain since a map of their location is saved in memory. Merging all fields and presenting an overall survey of the results allows a quick and efficient review of a sample. The map also produces accurate stereological measurements.

\section{Results}

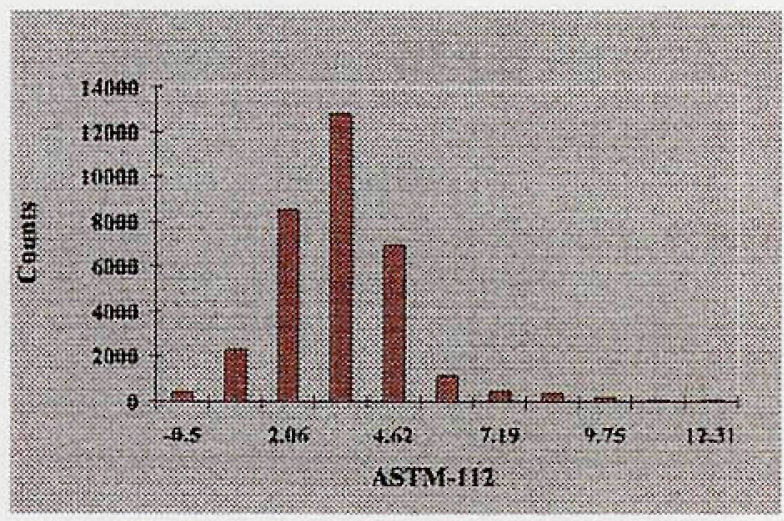

Fig.11 Cumulated statistics for ASTM-112
The color segmentation algorithm detects adjacent grains effectively over $\stackrel{\circ}{\stackrel{0}{0}}$ several samples. It is especially effective with noisy grains that would be barely 응 visible to a human operator. Only a few small grains were missed. Some? difficulties were encountered, however, when two adjacent grains had very similar $\vec{a}$ color or when large grains renlained heterogeneous cven after the morphological prefiltering. These cases led to a very poor color gradient.

Typical statistical results of the ASTM-112 measurement for an Al ingot are presented in figure 11 as a frequency distribution where each bin represents the grains that fall within a range of ASTM-112 measurements.

A correlation between two measurements, such as ASTM-112 and the orientation of the grain, can also be generated. Figure 12 presents this correlation $\frac{c}{\sigma}$ for a rolled aluminum sample having horizontal elongated grains. Clusters are $\frac{\overline{\bar{n}}}{\bar{\pi}}$ mainly grouped around 0 and 180 degree, as expected.

\section{Other applications}

The same segmentation algorithm was also tested on other similar applications for characterization of macro and microstructures seen with polarized light. Its robustness and generality seem very promising.

\section{Discussion}

The robustness of an algorithm is not the only factor that makes it a viable alternative to traditional methods. In order to make color segmentation usable in industry, speed is a key factor. Too many algorithms have been proposed without this consideration in mind. The system used for this project can analyze a field in less than 30 seconds even though it combines over 150 basic and advanced image analysis functions for each field. Recent optimizations and simplifications have lead to an analysis time of less than 15 seconds, allowing 200 fields to be processed in less than one hour.

Another important advantage of this approach is the ease with which this algorithm can usually be modified and optimized to accommodate samples in different conditions. The image analysis system used for this project has fulfilled all requirements and enabled us to obtain accurate results in an elegant and efficient manner.

\section{Acknowledgments}

The authors wish to express their sincere thanks to Vincent Fournier for is valuable advice during the development of the color morphological transforms, to Myriam Savard who tested the algorithms and 10 Louis Beauregard for his help in proofreading this paper.

1) Cigdem M. Bennett G.H.J., A Metallographic Examination of As-cast and As-processed Structures of C.P. Aluminum, Practical Metallography, Vol. XXIX, March 1992. 118-131

2) Starkey 3., Samantary A.K., Edge detection in petrographic images, Journal of Microscopy. Vol.172 Pl 3. December 1993.263-266

3) Breen E.J., Regression methods for automated colour image classification and thresholding, Journal of Microscopy, vol.174. Ft 1. April 1994, 23-30

4) Sarabi A., Aggarwal J.K, Segmentation of chromatic images Pattern Recognition, vol. 13, no.6. $1981,417-427$

5) Serra 3., Image Analysis and Mathematical Morphalogy, Academic Press, 1982, 610 p.6)

Serra 3., Image Analysis and Mathematical Morphology 2 : Theoretical Advances. Academic Press $1988,411 \mathrm{p}$

7) Beucher S., Segmentation dimages et morphologie mathematique, Thesis Doct. Ecole Nationale Supreure des Mines de Paris, $1990,295 \mathrm{p}$

8) Beucher S., Lantuejoul $C$., Use of watersheds in contour detection, Int. Workshop on Image Processing, CCET/IRISA, Rennes, France, 79

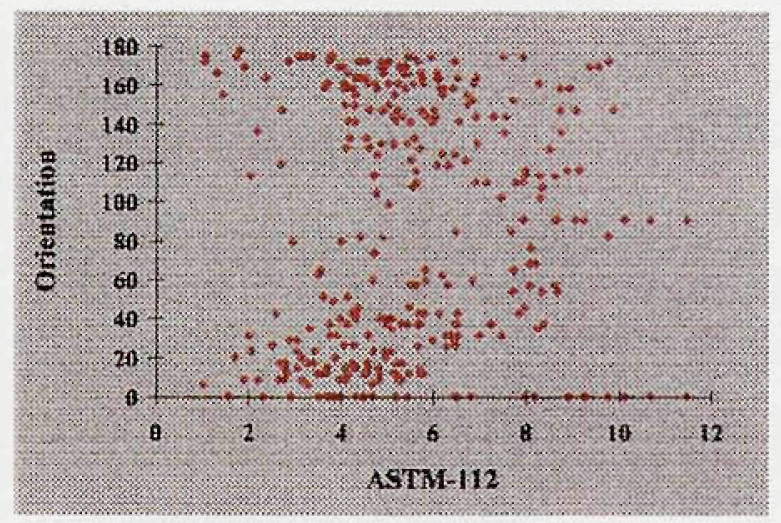

Fig.12 Correlation between orientation \& ASTM-112 


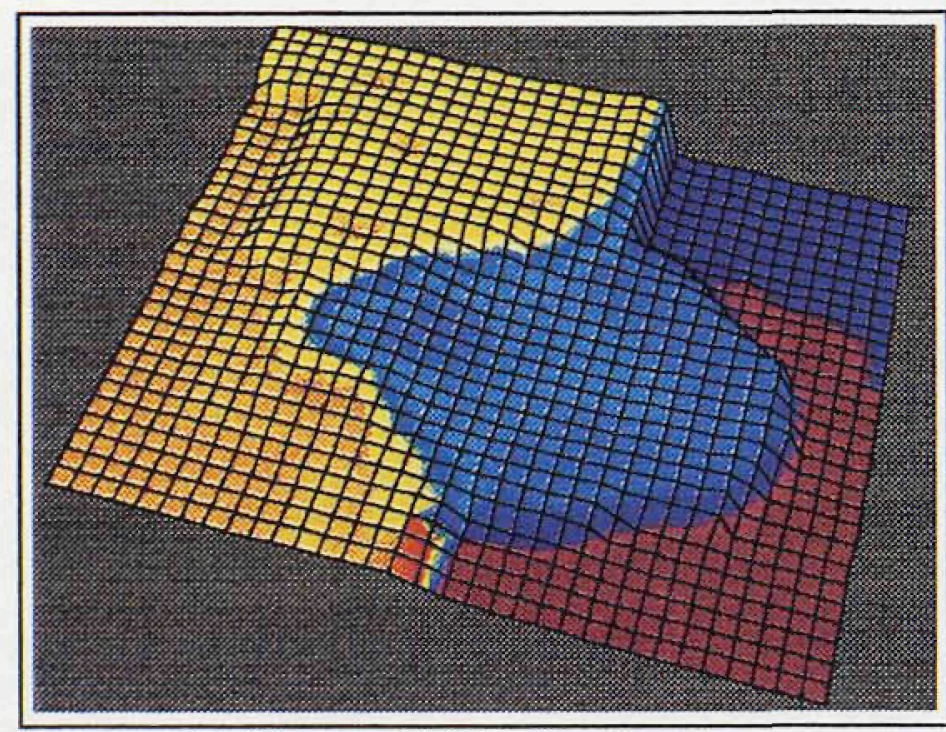

Fig.5 Color morphological closing

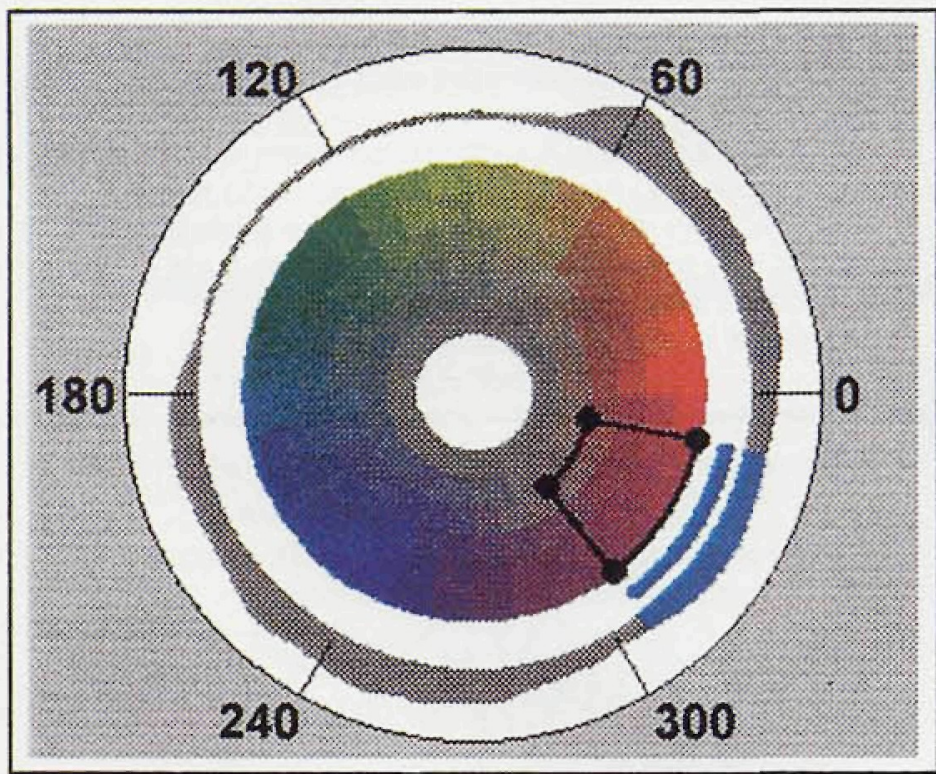

Fig.7 Hue and saturation thresholding

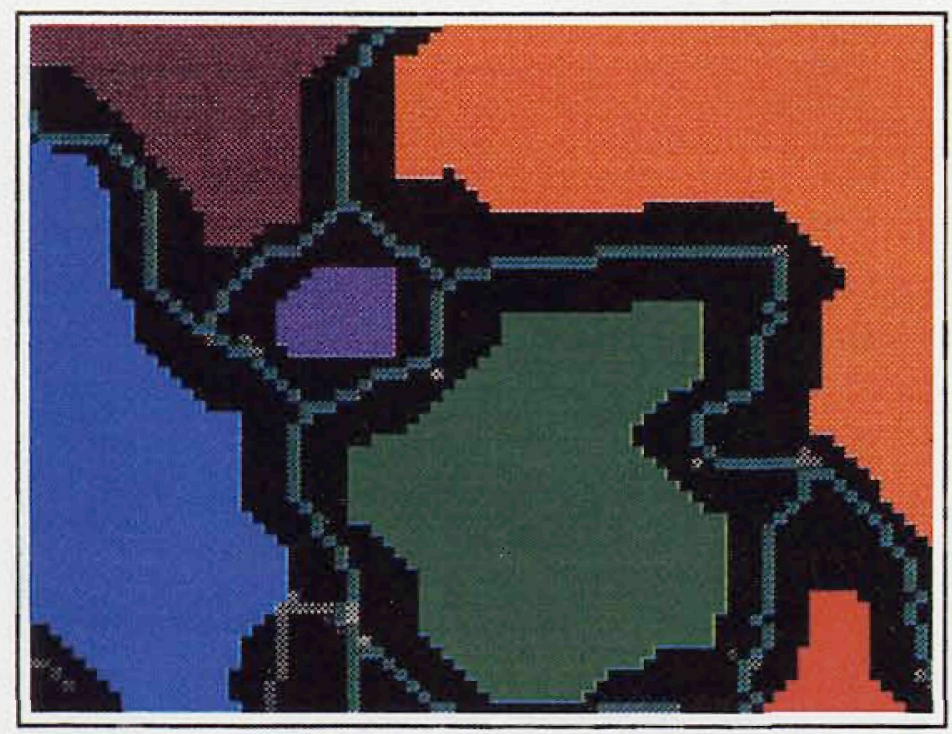

Fig.9 Coarse markers and color gradient

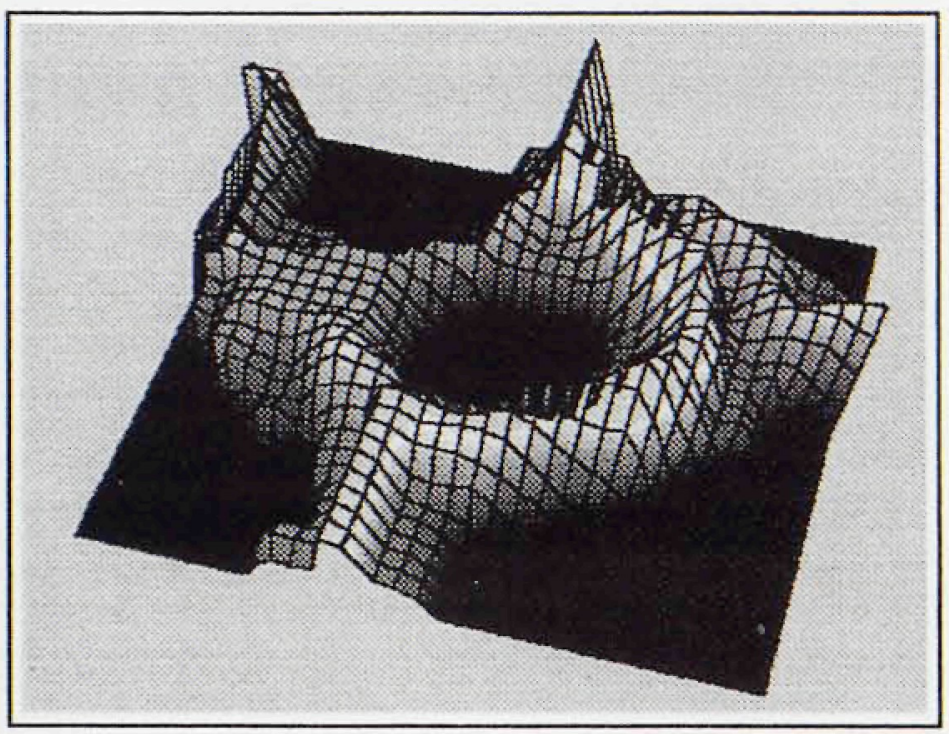

Fig.6 Filtered color gradient

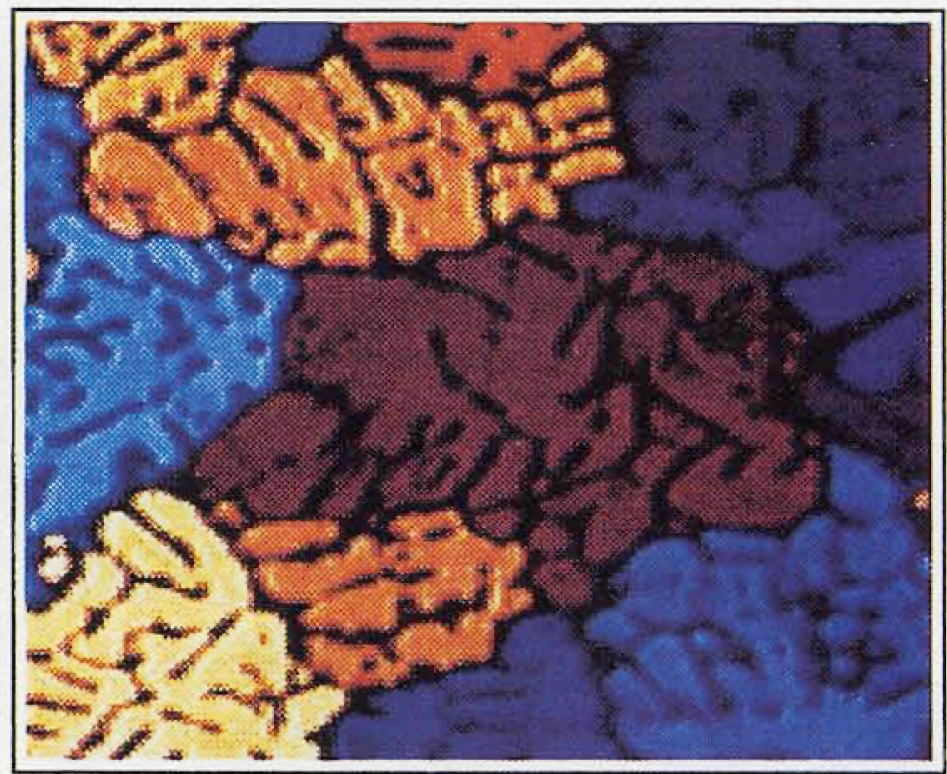

Fig.8 Grains detected after color thresholding

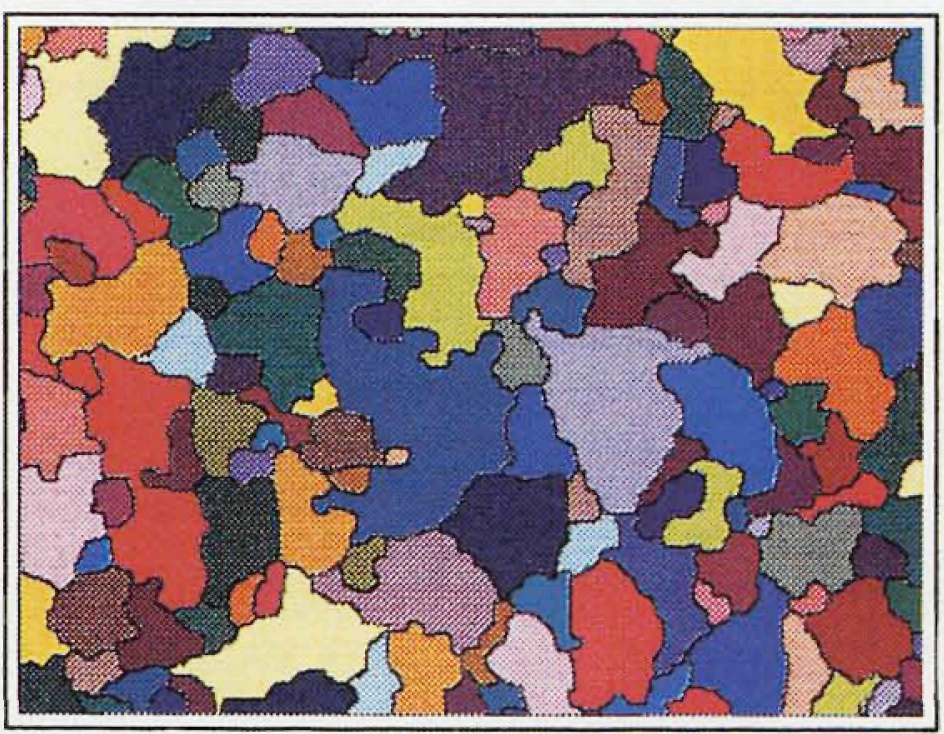

Fig.10 Final segmentation (labeled grains) 